\title{
Tratamiento Rehabilitador Estético-Oclusal con Resinas Compuestas en una Paciente con Mordida Profunda y Desgaste Severo
}

\author{
Aesthetic-Occlusal Rehabilitation Treatment with Composite \\ Resins in a Patient with Deep Bite and Dental Wear
}

\author{
Rony Christian Hidalgo-Lostaunau ${ }^{1,2}$
}

HIDALGO-LOSTAUNAU, R. C. Tratamiento rehabilitador estético-oclusal con resinas compuestas en una paciente con mordida profunda y desgaste severo. Int. J. Odontostomat., 14(1):73-80, 2020.

RESUMEN: La rehabilitación oral tradicional del paciente con mordida profunda y braquifacial puede ser muy compleja e invasiva, sin embargo, podría involucrar muchas especialidades para su resolución ideal. Un análisis integral estético-oclusal es necesario antes de empezar el tratamiento para hacer propuestas con el menor costo biológico, de tiempo y económico. La odontología adhesiva permite una propuesta aditiva y no invasiva como un tratamiento de mediano plazo o transitorio. Este artículo presenta un caso de una paciente de 46años de edad con mordida profunda anterior y desgaste dental severo en las caras palatinas de los dientes antero-superiores, sin disfunción temporomandibular, ni compromisos sistémicos. Se destaca la rehabilitación estética- oclusal con el mínimo compromiso biológico (ultra-conservadora), mediante restauraciones adhesivas semi-indirectas de resina compuesta con acompañamiento de 12 meses. Esta propuesta rehabilitadora con resinas compuestas representa una alternativa de tratamiento para el manejo del paciente con mordida profunda anterior. El control a un año de tratamiento demostró que se siguió una secuencia y resolución que aseguró los principios de la rehabilitación oral, al mismo tiempo, se procedió de la manera menos invasiva posible.

PALABRAS CLAVE: oclusión dental; estética dental; resina compuesta; desgaste dental, restauración dental.

\section{INTRODUCCIÓN}

Un tratamiento de rehabilitación completa de la boca pretende restaurar la forma y función del aparato masticatorio tan cerca de lo normal como sea posible con prótesis fijas (con o sin implantes), en la maxila y en la mandíbula (GPT-9, 2017). Entre las diversas situaciones clínicas que necesitan de una rehabilitación oral completa, el abordaje del desgaste dental severo o generalizado representa un reto diferente en la odontología contemporánea. La terapéutica podría ser enfocada primordialmente en la reconstrucción del tejido dental perdido, proveyendo buena estabilidad a largo plazo, función y estética (Ibsen et al., 1992; Schmidlin et al., 2009), sin necesariamente recurrir a un tipo de prostodoncia tradicional que implique mayor desgaste dentario para recubrir con coronas.

En la últimas dos décadas, se ha puesto énfasis en la conservación de tejidos, evitar el daño pulpar y el reforzar los tejidos dentales fragilizados, brindando tratamientos más duraderos con restauraciones directas o indirectas (Dietschi \& Spreafico, 1997; Magne et al., 2013). El uso de resinas compuestas directamente para restaurar denticiones severamente desgastadas fue descrito por Bevenius et al. (1994), proponiendo técnicas relativamente económicas y no invasivas, además de resultados estéticamente agradables.

La versatilidad que poseen las resinas compuestas radica en que nos permiten realizar distintos tipos de restauraciones dentales en situaciones donde otros materiales dentales no pueden sin prescindir de preparaciones dentarias, de manera que podemos abordar los desgastes dentales, erosiones o abrasiones oclusales, por ejemplo, con restauraciones de resina compuesta sin realizar ninguna preparación, -no invasividad- en la dentición que ya se encuentra desgastada. Su gran característica es que distribuyen mejor el estrés en su masa sin fracturarse prematuramen-

\footnotetext{
${ }^{1}$ Universidad Nacional Mayor de San Marcos, Unidad de Postgrado, Facultad de Estomatología, Lima, Perú.

${ }^{2}$ Universidad Científica del Sur. División de Rehabilitación Oral, Escuela de Odontología, Lima, Perú.
} 
te si están bien adheridas o cementadas (El Zohairy et al., 2003; Hamburger et al., 2014).

La posibilidad de manejar incrementos de la dimensión vertical (DV), nivelar planos oclusales (PO) y recrear la guía anterior con resinas compuestas ha sido probada y comprobada su longevidad a corto y mediano plazo (Al-Khayatt et al., 2013; Abduo \& Tennant, 2015; Mesko et al., 2016). Es así que se han desarrollado diversos protocolos y metodologías para el logro de estos y otros objetivos generales de la rehabilitación (Magne et al., 2007; Vailati \& Belser, 2008; Dietschi, 2009; Spreafico, 2010).

El objetivo del presente reporte de caso es presentar un protocolo simplificado, organizado en dos momentos clínicos: la rehabilitación de la guía anterior y la consolidación de la estabilidad posterior, ejemplificando que puede ser ejecutada una rehabilitación estética y oclusal de boca completa, con el mínimo compromiso biológico, empleando restauraciones adhesivas semi-indirectas de resina compuesta mediante el protocolo que será detallado.

\section{PRESENTACIÓN DEL CASO}

Una paciente de 46 años de edad, de sexo femenino acudió a la consulta solicitando un tratamiento no ortodóntico, debido a su inconformidad con su sonrisa. Las herramientas para el diagnóstico y planificación implicaron: examinación clínica, anamnesis, historia clínica, radiografías, modelos de estudio, montaje de modelos con arco facial en articulador semiajustable, encerado multipropósito (diagnóstico y planificación), fotografías extra-orales, intra-orales y para el diseño digital personalizado de la sonrisa (Hidalgo-Lostaunau, et al., 2014).

El análisis de las condiciones clínicas extra-orales e intra-orales reveló un desorden funcional de la oclusión por disminución de la dimensión vertical (proporción de Willis $56 / 50 \mathrm{~mm}$ ), mordida profunda anterior (95\%), ausencia de los dientes 24 y 36, signos severos de desgaste dental en las caras palatinas de los dientes anterosuperiores y múltiples restauraciones de amalgama dental y resina compuesta en los dientes posteriores (Fig. 1); el análisis estético-oclusal evidenciaba
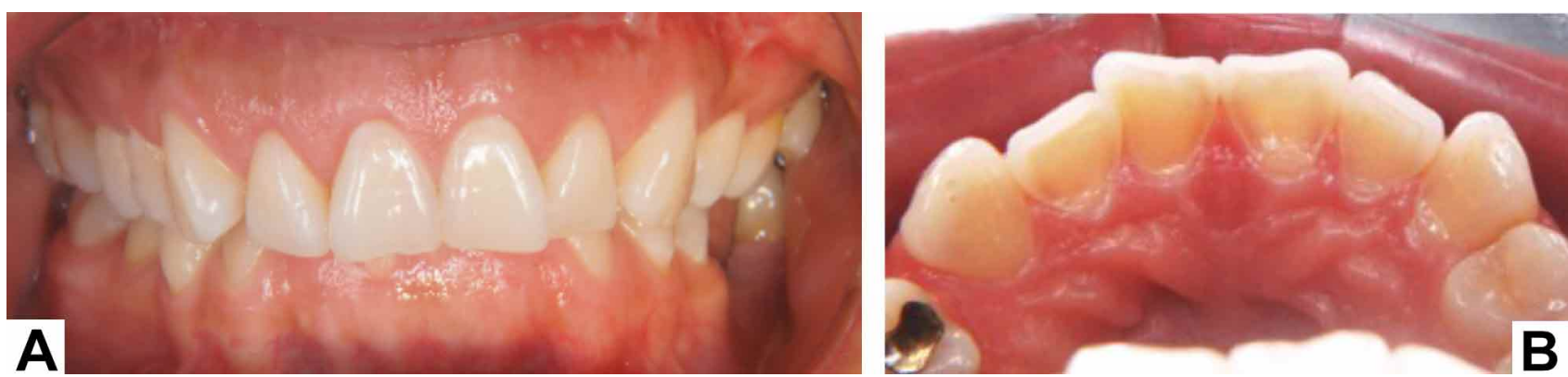

Fig. 1. Condición inicial con mordida profunda anterior (A). El desgaste severo en las caras palatinas de los incisivos y caninos superiores por bruxismo anterior $(B)$ no era advertida por la paciente hasta que se le mostraron sus fotos y modelos de estudio.

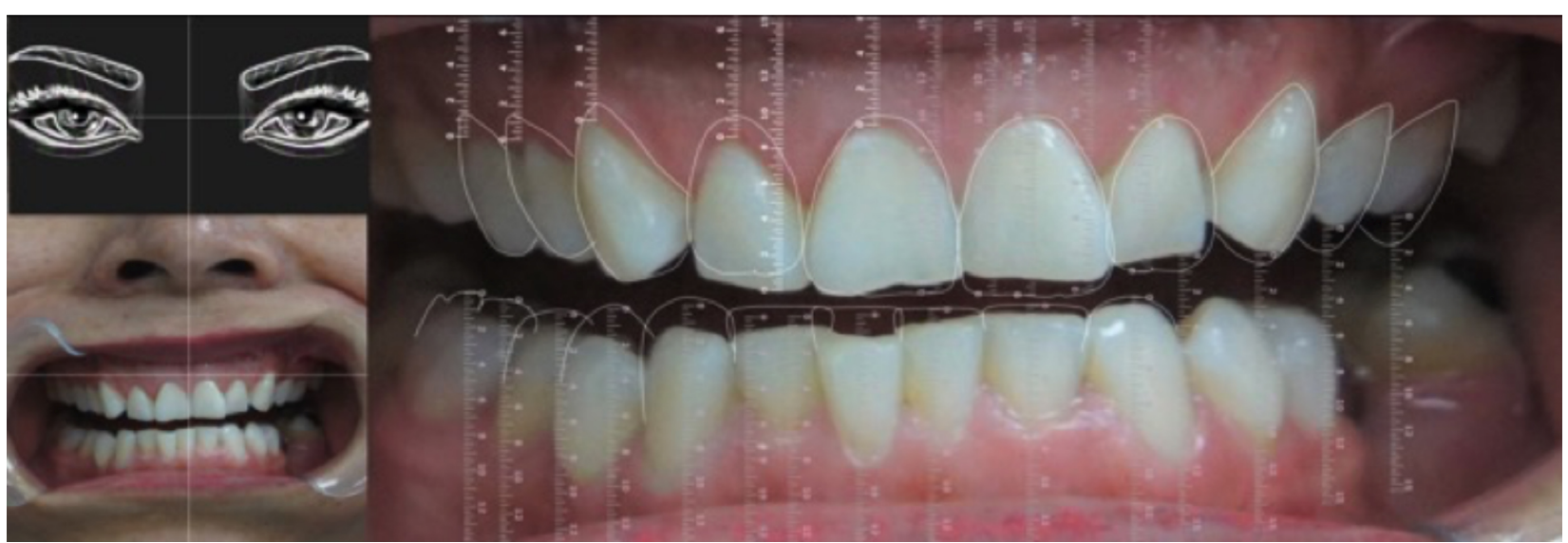

Fig. 2. Análisis de las condiciones intraorales en una fotografía digital de rostro completo en posición natural de la cabeza y diseño personalizado de la sonrisa para una comprensión de los posibles cambios estético-oclusales que permitan una mutua protección funcional entre dientes anteriores y posteriores. El milimetrado se consiguió a partir de aplicar la técnica del diseño personalizado de la sonrisa, CSD (Custom Smile Design), desarrollada por el autor el año 2011. 
una línea de la sonrisa y el plano oclusal desnivelado y canteado, un apiñamiento dental moderado, una curva de Spee de $4 \mathrm{~mm}$ y ausencia de la discrepancia funcional entre los tamaños de los incisivos centrales y laterales (Fig. 2).

Por fines didácticos el caso puede ser clasificado dentro de la Categoría I de Turner \& Missirlian (1984), por el excesivo desgaste y reducción de la dimensión vertical; como un caso Clase IV según The American College of Prosthodontists (2005) por su severidad en el compromiso del esquema oclusal y la necesidad de restablecer la dimensión vertical; o como un caso del Grupo III: A3P3 propuesto por Hidalgo-Lostaunau \& GanozaParedes (2013), según el nivel de complejidad pues requiere restablecer tanto el sector anterior para devolver la guía anterior perdida por el desgaste severo, como el posterior para nivelar los planos oclusales posteriores (POP) y fundamentalmente incrementar la DV.

Los objetivos de tratamiento fueron incrementar la dimensión vertical oclusal (DVO), nivelar los POP, estabilizar la oclusión, restructurar y otorgar funcionalidad a la guía anterior, para lo cual se presentaron dos propuestas: A) Tratamiento Ortodóntico Restaurador: donde se restauren únicamente los dientes desgastados (palatino e incisal de antero-superiores) y luego se procediese a un tratamiento de extrusión y nivelación de dientes posteriores, y B) Tratamiento Rehabilitador Estético-oclusal (A.R.T. por sus siglas en inglés): donde se traten por sectores los seis sextantes en un orden que permita evidenciar el incremento efectivo de la DV, el nivelamiento del plano oclusal y la optimización de la guía anterior por medio de restauraciones aditivas de resina compuesta.
La paciente se decidió por la segunda propuesta con restauraciones semi-indirectas de resina compuesta, entendiendo las ventajas (menos oneroso e invasivo, potencialmente reversible y reparable) y desventajas (rehabilitación con restauraciones de alrededor del $80 \%$ de longevidad en 8 años, pérdida de brillo, posibles desgastes o fracturas, necesidad de controles de mantenimiento) de las mismas (Muts, et al., 2014; Loomans, et al., 2017). El tratamiento se condujo mediante la siguiente secuencia metodológica:

Planificación del encerado multipropósito en los modelos montados en ASA. Los modelos montados en ASA en relación céntrica permitieron una ganancia vertical anterior de $4 \mathrm{~mm}$ y anteroposterior de manera que se proyecte el sobrepase vertical en un máximo del $60 \%$ de encubrimiento de los incisivos inferiores (loannidou, et al., 1999; John, et al., 2002). Las indicaciones para ello fueron encerar los incisivos y caninos inferiores hasta nivelarlos según la propuesta del diseño digital (Fig. 2) y la relación labio-dental luego de la fonación, logrando una adecuada parábola del arco, y después encerar las caras palatinas de los incisivos y caninos superiores de manera que por vestibular se evidenciara el incremento siguiendo las medidas sugeridas en el diseño digital personalizado.

Evaluado el espacio interoclusal efectivo entre los dientes posteriores y estando de acuerdo con la relación entre el incremento de la DV con el acople y la guía anterior lograda (Fig. 3A), en una segunda fase se mandaron a encerar los dientes postero-superiores primero y postero-inferiores después. Teniendo énfasis en la indicación del nivelamiento del plano oclusal canteado (evidencia-

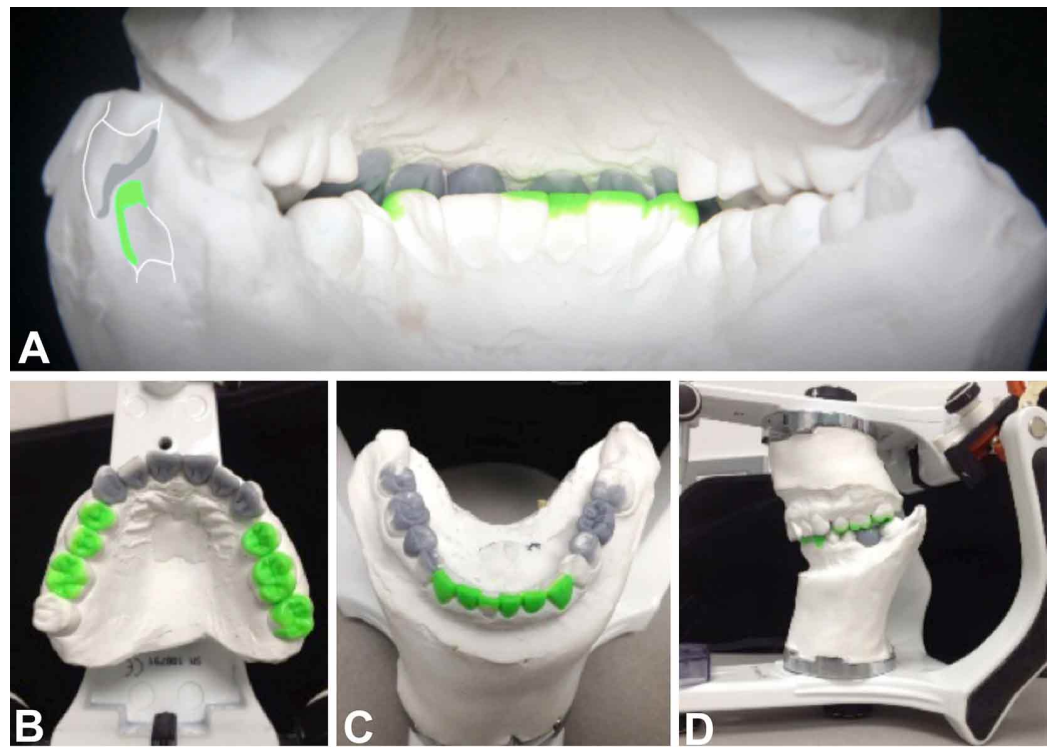

Fig. 3. Encerado de los sectores anteriores (inferior: vestíbulo-incisal, y superior: palato-incisal) y relación de acople anterior evaluada desde una perspectiva lingual (A). Encerado de los sectores posteriores superiores primero (B) y luego los postero-inferiores (C). Hasta cumplir con una curva de Spee levemente marcada y generar estabilidad oclusal por contactos equilibrados (D). 
do en la fotografía de rostro y el diseño digital bidimensional) (Fig. 2), se indicó adicionar cera en oclusal y a nivel de cúspides guía superiores izquierdas según las sugerencias del diseño digital orientándonos por la línea ocluso-maxilar; luego se indicó encerar los dientes postero-inferiores hasta lograr oclusión con una ligera curva de compensación. (Fig. 3B-3D).

Completado el encerado de diagnóstico, se explicó el plan de tratamiento a la paciente y siguiendo su petición respecto a la técnica que emplearíamos para confeccionar sus restauraciones, se procedió a retirar la cera de palatino de los antero-superiores por medio de un vaporizador, para luego aislar el yeso y confeccionar las carillas palatinas (Vailati, 2017), de resina compuesta Z250XT (3M-ESPE, Alemania) y pigmentos Kerr Color+ (Kerr Corporation, USA) con una técnica semi-indirecta a mano alzada, cotejando la guía anterior y las correctas desoclusiones.
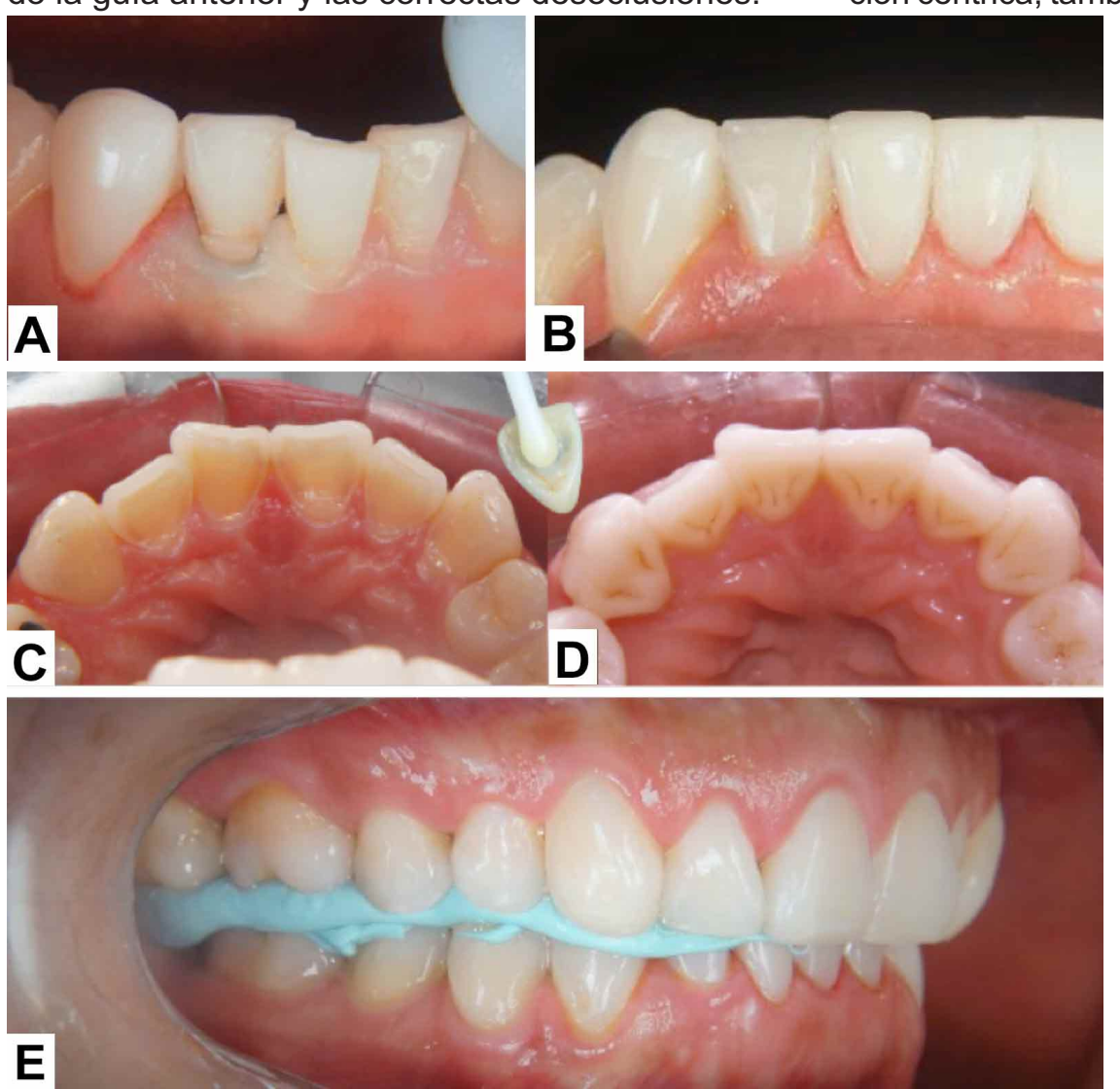

Fig. 4. Durante el aislamiento relativo se evidenciaron lesiones de abfracción subgingivales, la cuales fueron restauradas y selladas con las carillas vestibulares inferiores de los dientes $31,32,31,42,43$ (4A-B). Las carillas palatinas fueron cementadas con resina compuesta de alta carga, al mismo tiempo la prolongación incisal sirvió para nivelar el plano oclusal anterior (4C-D). El registro intermaxilar fue del espacio interoclusal efectivo logrado con el incremento de la DV que se planificó desde el encerado multipropósito inicial $(3 \mathrm{~A}, 4 \mathrm{E})$.
Restauración Intraoral de la Guía Anterior. Para realizar las restauraciones del sector antero-inferior predeciblemente, se tomaron llaves de silicona pesada Z-Labor (Zhermack, Liechtenstein) de los aspectos lingual y vestibular del encerado anteriormandibular. Con la llave lingual se llevó a cabo una resina directa en incisal del diente 41 , y se tomó su posición vestibularizada como guía para las restauraciones de resina compuesta tipo carillas vestíbuloincisales con resina compuesta Filtek Z250Xt (3MESPE, Alemania), en los dientes $33,32,31,42,43$, con una técnica indirecta-directa (Bueno, et al., 2015) para reducir el sobrepase horizontal y alinear la parábola del arco inferior (Fig. 4A-4B). Luego se cementaron las carillas palatinas de 13,12,11,21,22,23 con resina fluida Estelite FlowQuick (Tokuyama Dent Corp., Japón), se pulieron los márgenes con fresas multilaminadas, tiras de lija y discos de pulido (Fig. 4C-4D). Se cotejó la oclusión y desoclusiones en relación céntrica, también comprobó la adecuada fonación y la apariencia estética en estática y dinámica, luego se tomó un registro intermaxilar (Fig 4D) con Blu-Mousse (Parkell Inc. USA), impresiones completas superior e inferior con silicona por adición Express XT (3M-ESPE, Alemania), se confeccionaron modelos de yeso tipo IV y se articularon nuevamente. Se aislaron los dientes posteriores de los modelos con Rubber-Sep (Kerr Corporation, USA) y en el modelo superior se confeccionaron con resina compuesta Z250XT y tintes Kerr Color+, las restauraciones oclusales, se adaptaron y pulieron antes de empezar las restauraciones inferiores de la misma manera. Se chequeó la oclusión, desoclusiones y se procedió a arenarlas internamente para dejarlas listas para su cementación (Fig. 5) en la segunda sesión clínica.

Cementado de las Restauraciones Posteriores. Estas restauraciones no necesitaron de ningún tipo de preparación protocolar en los dientes pilares, sólo se eliminaron aristas de los sustratos de esmalte y se asperizaron las restauraciones de resina compuesta 

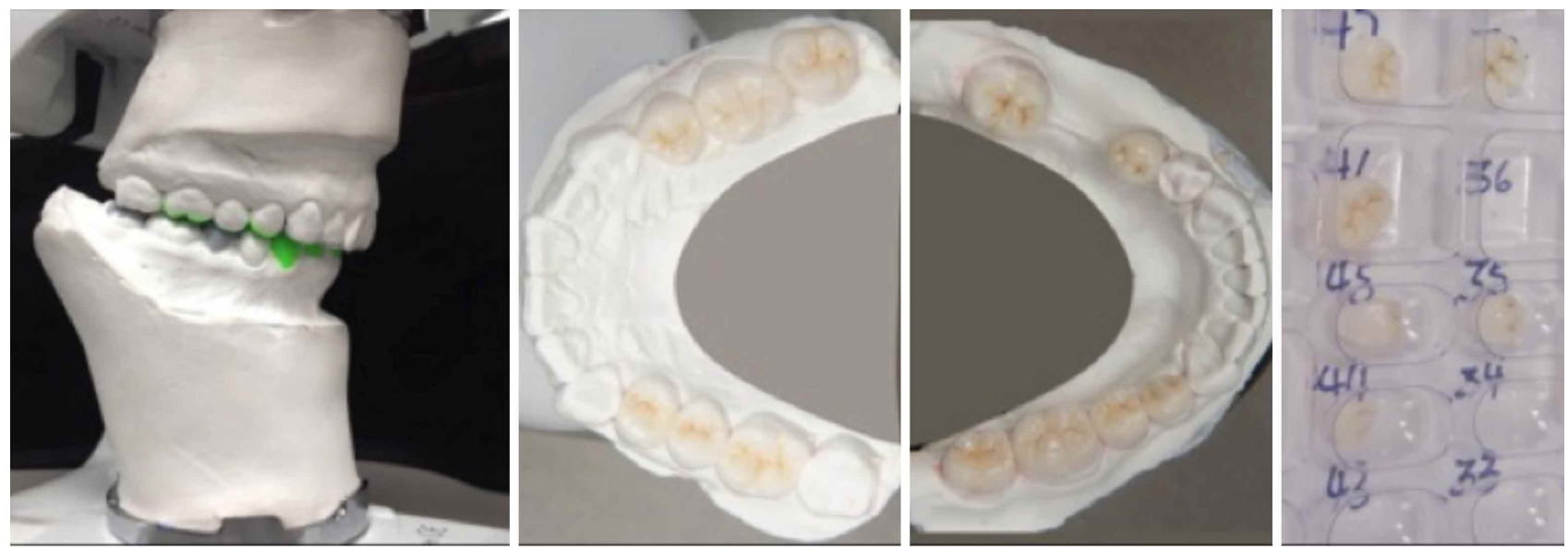

Fig. 5. Restauraciones semi-indirectas de resina compuesta pulidas y listas para su cementación por sextantes. Su organización en estuches es muy importante para su correcta manipulación durante los procesos de limpieza, silanización y cementación.
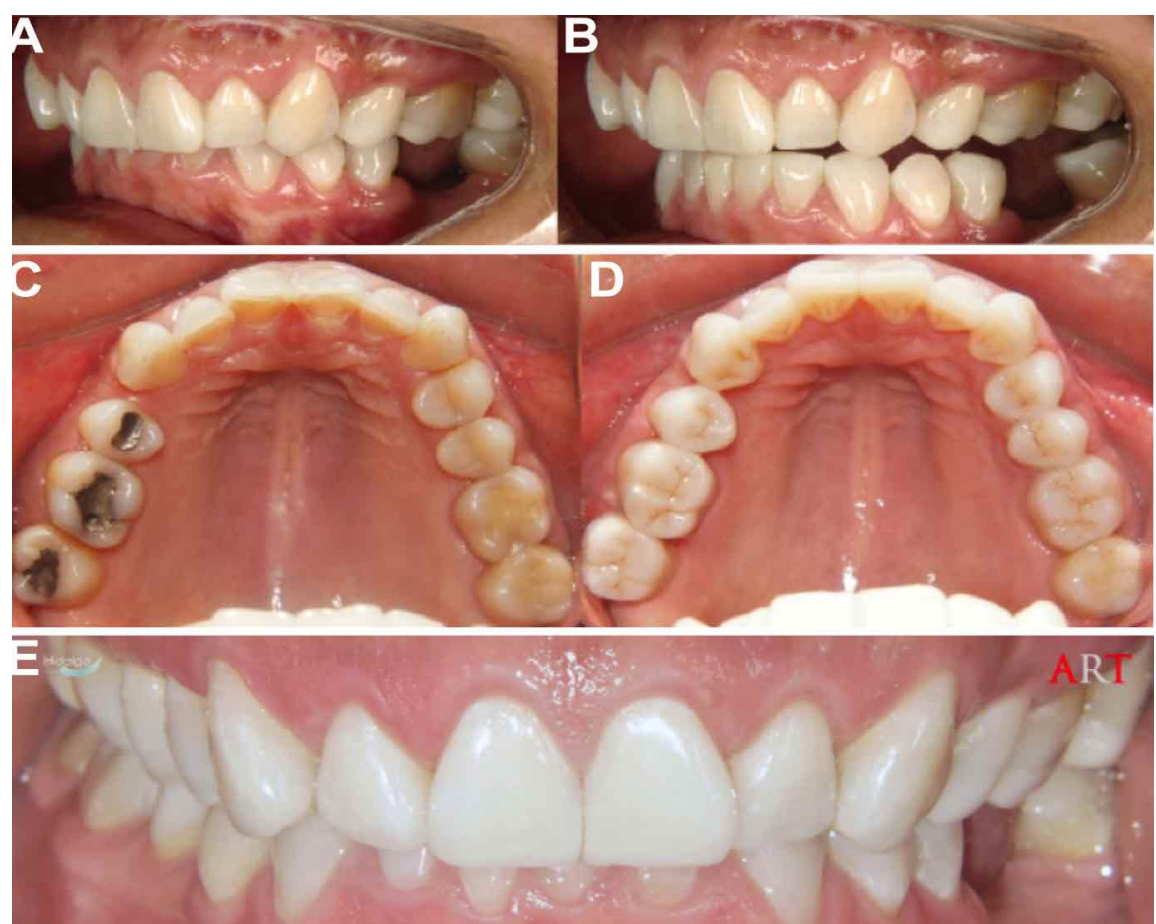

\section{2 meses}

Dic2018

Fig. 6. Movimiento funcional de guía canina del lado izquierdo (A,B). Arcada superior completamente restaurada y cerrando el diastema entre los dientes 17 y 16 , como entre 15 y $13(C, D)$. Control 1 año (E), compárese con la Fig. 1A.

en buen estado (las obturaciones de amalgama fueron previamente retiradas y reemplazadas por resinas compuestas directas). Con estas carillas oclusales (de 0,6 a 1,5 mm en promedio en los diferentes sextantes) se consolidó la DV, se nivelaron los POP y se generó una leve curva de Spee. Fueron cementadas primero los sextantes superiores desde la más distal hacia la más mesial, y luego las inferiores de la misma manera, se empleó el adhesivo Single Bond Universal (3M-ESPE, Alemania) más el cemento RelyX Ultimate (3M-ESPE, Alemania). Se pulieron las terminaciones mar- ginales, se chequeó la oclusión céntrica, las desoclusiones y se ensayó la masticación (Fig. 6).

Una vez finalizado el tratamiento, la paciente acudió a sus controles respectivos a la semana, al mes y al año. En dichos periodos fueron observados resultados favorables en su adaptación, satisfacción estética y funcional referidas por la paciente.

\section{DISCUSIÓN}

El desgaste dental es generalmente un proceso fisiológico; sin embargo, también puede ser una condición patológica asociada con el bruxismo y los procesos de erosión en los cuales la cantidad de pérdida de tejido dentario es atípica para la edad del paciente; esta pérdida a menudo causa problemas funcionales, síntomas de malestar o dolor, y alteraciones estéticas (Ergun \& Yucel, 2014).

La rehabilitación oral completa de pacientes con mordida profundizada, desgastes dentarios severos, edentulismos parciales, requerimientos de nivelamiento de planos oclusales y manejo de la 
DV ha sido clasificada en la literatura odontológica como la más compleja, según The American College of Prosthodontics (McGarry et al., 2004).

En el presente relato de caso, la superficie palatina de los dientes antero-superiores presentaban un desgaste severo, que conjugado con la disminución de la DV evidenciada en el rostro y la excesiva mordida profunda intraoralmente, condujeron a una propuesta de tratamiento para el restablecimiento de la DV oclusal. La cual fue lograda por medio del manejo de los sectores anteriores y posteriores con restauraciones de resina compuesta semi-indirectas, que pueden ser confeccionadas por el operador o por el técnico en prótesis dental con resinas compuestas para uso clínico o laboratorial.

Como menciona Saeidi-Pour et al. (2017) el encerado de diagnóstico proporciona la asistencia precisa para determinar un nuevo plano oclusal y la DVO, por lo que consideramos es una herramienta fundamental que procuramos traducir hacia las restauraciones de resina con diferentes técnicas, en la ejecución de una rehabilitación oral completa. Lo innovador en este caso fue el retiro de la cera de palatino de anteriores, estando los modelos montados con la DV determinada y la propuesta funcional completa por los encerados de los dientes posteriores, así es que se confeccionaron con resina compuesta las carillas palatinas en los mismos modelos originales, para luego retirarlas y cementarlas en boca (Fig. 4C-D), una vez que los antero-inferiores ya habían sido restaurados (Fig. 4A-B); finalmente realizamos las restauraciones posteriores prácticamente sin preparaciones (tallados) dentales.

Los conceptos de rehabilitación completa tradicional incluyen principalmente realizar coronas de porcelana fundida a metal o recubrimientos cerámicos. Este tratamiento no solo es invasivo, sino que también requiere mucho tiempo y es costoso. Particularmente en los países con pacientes que pagan por cuenta propia, las modalidades de tratamiento menos costosas serían muy interesantes, y las restauraciones de resina compuesta serían una valiosa opción para la rehabilitación estética y oclusal (Schmidlin et al.), a la vez que han sido una alternativa perfectamente aceptada al igual que las cerámicas dentales (Mesko et al.; Loomans et al.).

La longevidad de las restauraciones directas de resina compuesta en dientes anteriores ha sido probada en múltiples estudios (Abduo \& Tennat; Al-Khayatt et al.; Mesko et al.; Hamburger et al.; Hemmings et al.), siendo una alternativa poco onerosa, funcional y mínimamente invasiva para el manejo de denticiones severamente gastadas. Así mismo, las carillas palatinas fueron propuestas desde que su eficacia como tratamiento conservador, no invasivo y aditivo fue comprobada en la técnica de Dahl (Beddis, et al.; Gulamali et al.; Magne et al.; Vailati \& Belser), y en investigaciones de seguimiento como el estudio de Erosión de Ginebra (Vailati et al.; Vailati).

La condición oclusal con la mordida posterior abierta luego de la resolución ultra-conservadora de los sectores anteriores permaneció por dos días. Consideramos que esta situación corresponde a una condición semejante al empleo de un deprogramador neuromuscular, el cual consolida la posición céntrica de la mandíbula, y como asevera Poyser et al. (2007), las restauraciones de resina actúan como un dispositivo ortodóntico fijo y los dientes están protegidos por propiocepción en los ligamentos periodontales mientras el paciente se adapta. La confianza en este procedimiento se basa en el trabajo realizado originalmente por Anderson en 1962 quien mostró que los pacientes se adaptan fácilmente a los cambios planificados en la oclusión (Redman, et al., 2003).

La cementación de las restauraciones posterosuperiores y postero-inferiores se realizaron al tercer y cuarto día de consulta, respectivamente. Estas restauraciones finalmente consolidaron la DVO y los POP (curva de Spee y Wilson) logrando disclusión canina (oclusión mutuamente protegida), esquema oclusal donde estos materiales han probado su factibilidad y comprobado su longevidad (Abduo \& Tennat; Dietschi; Hamburger et al., 2011).

El protocolo empleado, denominado: Tratamiento Rehabilitador Estético-Oclusal empleado en este caso, difiere en la secuencia del abordaje, tanto laboratorial y clínica de otras metodologías previamente difundidas en la literatura odontológica (Dietschi; Magne et al.; Spreafico; Vailati \& Belser), sin embargo, los objetivos generales de la rehabilitación son perseguidos por todos indistintamente del orden protocolar propuesto.

El A.R.T. (por sus siglas en inglés; AestheticOclusal Rehabilitation Treatment) aprovecha la versatilidad de las resinas compuestas, para realizar distintos tipos de restauraciones en escenarios donde otros materiales dentales no podrían sin dejar de ser sustractivos. Un abordaje no invasivo y aditivo en re- 
sinas compuestas se pudo ejecutar en este caso, como una alternativa de tratamiento en una paciente con mordida profunda anterior; la odontología adhesiva en sí y un manejo preciso de los fundamentos de la oclusión nos permiten proponer nuevas opciones de tratamiento, con el mínimo costo biológico, de tiempo y económico, donde generalmente se podría realizar una odontología más tradicional.

Las resinas compuestas actualmente representan una opción restauradora para los dientes posteriores, técnica y clínicamente comprobada, proveyendo un buen y predecible resultado a largo plazo, sin embargo, algunos investigadores han encontrado que los resultados más favorables están muy relacionados a la composición específica de las resinas compuestas empleadas (Abduo \& Tennat; Dietschi \& Spreafico, 1998; El Zohairy et al.; Vailati \& Belser).

Podría sugerirse emplear el protocolo del A.R.T. con otras alternativas en biomateriales, como por ejemplo cerámicas híbridas o el uso de tecnologías como el Cad-Cam para la confección de las piezas protésicas, siempre y cuando sea una odontología preponderantemente aditiva o mínimamente invasiva donde el diagnóstico y adecuado manejo de la DVO sea el primer paso que conduzcan a restauraciones planificadas hacia los objetivos fundamentales de la rehabilitación. Controles de mantenimiento y estudios de longevidad en diferentes situaciones clínicas han de continuarse para determinar los alcances máximos de este tipo de rehabilitaciones, por el momento se conoce que los porcentajes de éxitos de estas restauraciones oscilan entre el 90-95 \% (3-5años) y el 80-85 $\%$ (7-8años) con un rango de falla anual del $2 \%$ (AlKhayatt et al., ; Mesko, et al.; Abduo \& Tennat; Gulamali, et al.; Hamburger et al., 2011; Loomans, et al.; Muts, et al.; Poyser et al.).

El control realizado a los 12 meses de ejecutado del tratamiento reveló las condiciones de las restauraciones de resina compuesta, que no presentaron desgaste aparente, signos de fractura, cambios de color, ningún deterioro clínica o radiográficamente evidente, con altísimo grado de satisfacción de la paciente, sobre todo por que pudimos evitar tallados protésicos tradicionales. El sobrepase horizontal y vertical, el acople, la guía anterior, el nivelamiento del plano oclusal, la mejora en el DVO gracias a las restauraciones realizadas, otorgaron comodidad y funcionalidad al sistema estomatognático de la paciente que refirió su alto nivel de satisfacción y comodidad. Es importante seguir registrando en controles sucesivos los cambios clínicos y radiográficos que puedan manifestarse para tener una noción más completa de los alcances de este tratamiento rehabilitador.

Teniendo en cuenta esto, la rehabilitación con resinas compuestas es sin duda más conservadora que las preparaciones dentarias tradicionales para restauraciones indirectas parciales o completas y los datos parciales hasta la fecha, muestran que esta opción ofrece buenos resultados clínicos y pacientes muy satisfechos (Hamburger et al., 2011; Mesko, et al.; Loomans, et al.).

\section{AGRADECIMIENTOS}

A la Srta. Donia Pedraza Huamán, TPD., por su excelencia en la elaboración de los encerados del caso, como también en la confección de las carillas palatinas y restauraciones postero-inferiores.

A la Dra. Evelyn Álvarez Vidigal, CD, Esp, Ms, $\mathrm{PhD}$ por su revisión y motivación para redactar este reporte de caso.

HIDALGO-lostaunAU, R. C. Aesthetic-occlusal rehabilitation treatment with composite resins in a patient with deep bite and dental wear. Int. J. Odontostomat., 14(1):7380, 2020.

ABSTRACT: The traditional oral rehabilitation of the patient with deep bite and brachifacial can be very complex and invasive, however, a number of treatments could be considered to improve rehabilitation. A comprehensive aesthetic-occlusal analysis is necessary prior to starting treatment in order to consider the best procedure, time involved, and economic cost. Adhesive dentistry allows an additive and non-invasive proposal as a medium-term or transitory treatment. A case of a 46-year-old patient is presented, with anterior deep bite and severe dental wear on palatal surfaces of the antero-superior teeth, without temporomandibular dysfunction, or systemic compromises. The objective was to emphasize the aesthetic and occlusal rehabilitation with the least biological commitment (ultraconservative), through semi-indirect adhesive restorations of composite resin with 12 months follow-up. This restorative proposal with composite resins represents an alternative treatment for patient management with anterior deep bite. Control at one year of treatment showed that a sequence and resolution was followed, that ensured adequate oral rehabilitation in the least invasive manner possible.

KEY WORDS: dental occlusion; dental esthetic; composite resin, tooth wear, dental restoration. 


\section{REFERENCIAS BIBLIOGRÁFICAS}

Abduo, J. \& Tennant, M. Impact of lateral occlusion schemes: A systematic review. J. Prosthet. Dent., 114(2):193-204, 2015.

Al-Khayatt, A. S.; Ray-Chaudhuri, A.; Poyser, N. J.; Briggs, P. F.; Porter, R. W.; Kelleher, M. G.; et al. Direct composite restorations for the worn mandibular anterior dentition: a 7-year follow-up of a prospective randomized controlled split-mouth clinical trial. J. Oral. Rehabil., 40(5):389-401, 2013.

Anderson, D. J. Tooth movement in experimental malocclusion. Arch. Oral. Biol. 7(1): 7-15, 1962.

Beddis, H. P.; Durey, K.; Alhilou, A. \& Chan, M. F. W. Y. The restorative management of the deep overbite. Br. Dent. J., 217:509-15, 2014.

Bevenius, J.; Evans, S. \& L'Estrange, P. Conservative management of erosion-abrasion: a system for the general practitioner. Aust. Dent. J., 39(1):4-10, 1994.

Bueno, A. L. N.; Pupo, Y. M.; Araujo, F. O. \& Kubitski, M. Matriz Estética Personalizada (MEP): abordagem ultraconservadora e previsível para confecção de facetas semidiretas. Rev. Dental Press. Estét., 12(2):99-110, 2015.

Dietschi, D. The use of composite in severe abrasion and erosion cases. Aust. Dent. Pract., 132-4, 2009.

Dietschi, D. \& Spreafico, R. Adhesive Metal-Free Restorations: Current Concepts for the Esthetic Treatment of Posterior Teeth. Chicago, Quintessence Publishing, 1997.

Dietschi, D. \& Spreafico, R. Current clinical concepts for adhesive cementation of tooth-colored posterior restorations. Pract. Periodont. Aesthet. Dent., 10(1):47-54, 1998.

El Zohairy, A. A.; De Gee, A. J.; Mohsen, M. M. \& Feilzer, A.J. Microtensile bond strength testing of luting cements to prefabricated CAD/CAM ceramic and composite blocks. Dent. Mater., 19(7):57583, 2003.

Ergun, G. \& Yucel, A. S. Full-Mouth Rehabilitation of a Patient with Severe Deep Bite: A Clinical Report. J. Prosthodont., 23(5):40611, 2014.

GPT-9. The Academy of Prosthodontics Foundations. The Glossary of Prosthodontics Terms. Edition Nine. J. Prosthet. Dent., e1-e104, 2017. Disponible en: https://www.academyofprosthodontics.org/_Library/ ap_articles_download/GPT9.pdf

Gulamali, A. B.; Hemmings, K. W.; Tredwin, C. J. \& Petrie, A. Survival analysis of composite Dahl restorations provided to manage localised anterior tooth wear (ten year follow-up). Br. Dent. J., 211:E9, 2011.

Hamburger, H. T.; Opdam, N. J. M.; Bronkhorst, E. M. \& Huysmans, J. $M$. Indirect restorations for severe tooth wear: Fracture risk and layer thickness. J. Dent., 42(4):413-8, 2014.

Hamburger, J.T.; Opdam, N. J.; Bronkhorst, E. M.; Kreulen, C. M.; Roeters, J. J. \& Huysmans, M. C. Clinical performance of direct composite restorations for treatment of severe tooth wear. J. Adh. Dent., 13:58593, 2011.

Hemmings, K. W.; Darbar, U. R. \& Vaughan, S. Tooth wear treated with direct composite restorations at an increased vertical dimension: results at 30 months. J. Prosthet. Dent., 83:287-93, 2000.

Hidalgo-Lostaunau, R.C.; de Oliveira, G.G.; Cueva-Cueto, M.; VillalobosTinoco, J. \& Flores-Reyes, J. C. Desenho digital do sorriso. En: Miyashita, E.; de Oliveira, G.G. Odontologia Estética: os desafíos da clínica diaria. São Paulo, Napoleão, 2014.

Hidalgo-Lostaunau, R. C. \& Ganoza-Paredes, P. El diseño personalizado de sonrisa y el abordaje estético-oclusal. Parte II. Rev. APCD. Estet., 1(3):342-55, 2013.

Ioannidou, I.; Gianniou, E.; Koutsikou, T.; \& Kolokithas, G. Quantitative description of overjet and overbite and their relationship with the craniofacial morphology. Clin. Orthod. Res., 2(3):154-61, 1999.

Ibsen, R. L. \& Ouellet, D. F. Restoring the worn dentition. J. Esthet. Dent., 4(3):96-101, 1992.

John, M. T.; Hirsch, C.; Drangsholt, M. T.; Mancl, L. A. \& Setz, J. M. Overbite and overjet are not related to self-report of temporomandibular disorder symptoms. J. Dent. Res., 81(3):164-9, 2002.
Loomans, B.; Opdam, N.; Attin, T.; Bartlett, D.; Edelhoff, D.; Frankenberger, R.; Benic, G.; Ramseyer, S.; Wetselaar, P.; Sterenborg, B.; Hickel, R.; Pallesen, U.; Mehta, S.; Banerji, S.; Lussi, A. \& Wilson, N. Severe Tooth Wear: European Consensus Statement on Management Guidelines. J. Adhes. Dent., 19:111-9, 2017

Magne, P.; Magne, M. \& Belser, U. C. Adhesive restorations, centric relation, and the Dahl principle: minimally invasive approaches to localized anterior tooth erosion. Eur. J. Esthet. Dent., 2(3):260-73, 2007.

McGarry, T. J.; Nimmo, A.; Skiba, J. F.; Ahlstrom, R. H.; Smith, C. R.; Koumijan, J. H. \& Guichet, G.N. American College of Prosthodontics. Classification system for the completely dentate patient. J. Prosthodont., 13(2):73-82, 2004

Mesko, M. E.; Sarkis-Onofre, R.; Cenci, M. S.; Opdam, N. J.; Loomans, B. \& Pereira-Cenci, T. Rehabilitation of severely worn teeth: A systematic review. J. Dent., 48:9-15, 2016.

Muts, E. J.; van Pelt, H.; Edelhoff, D.; Krejci, I. \& Cune, M. Tooth wear: a systematic review of treatment options. J. Prosthet. Dent., 112(4):7529, 2014

Poyser, N. J.; Briggs, P. F. A.; Chana, H. S.; Kelleher, M. G. D.; Porter, R. W. J. \& Patel, M. M. The evaluation of direct composite restorations for the worn mandibular anterior dentition-Clinical performance and patient satisfaction. J. Oral. Rehab., 34(5):361-76, 2007.

Redman, C. D.; Hemmings, K. W. \& Good. J. A. The survival and clinical performance of resin-based composite restorations used to treat localized anterior tooth wear. Br. Dent. J., 194:566-72, 2003.

Saeidi-Pour, R.; Engler, M. L. P. D.; Edelhoff, D.; Prandtner, O.; Frei, S. \& Liebermann, A. A patient-calibrated individual wax-up as an essential tool for planning and creating a patient-oriented treatment concept for pathological tooth wear. Int. J. Esthet. Dent., 13(4):476-92, 2017.

Schmidlin, P. R.; Filli, T.; Imfeld, C.; Tepper, S. \& Attin, T. Three-year evaluation of posterior vertical bite reconstruction using direct resin composite--a case series. Oper. Dent., 34(1):102-8, 2009.

Spreafico, R. C. Composite resin Rehabilitation of Eroded Dentition in a Bulimic Patient: a case report. Eur. J. Esthet. Dent., 5(1):28-48, 2010.

Turner, K. A. \& Missirlian, D. M. Restoration of the extremely worn dentition. J. Prosthet. Dent., 52(4):467-74, 1984.

Vailati, F. Composite palatal veneers to restore a case of severe dental erosion, from minimally to non invasive dentistry: a 5-year follow-up case report. It. J. Dent. Med., 2(1):24-34, 2017.

Vailati, F. \& Belser, U. C. Full-mouth adhesive rehabilitation of a severely eroded dentition: the three-step technique. Part 1-2-3. Eur. J. Esthet. Dent., 3(1):30-44, 2008.

Vailati, F.; Gruetter, L. \& Belser, U. C. Adhesively restored anterior maxillary dentitions affected by severe erosion: up to 6-year results of a prospective clinical study. Eur. J. Esthet. Dent., 8(4):506-30, 2013.

Dirección de correspondencia:

Rony Christian Hidalgo Lostaunau

Jr. Pedro de Candia \#407, Valle Hermoso de Monterrico

Santiago de Surco

Lima 33

PERÚ

Email: contacto@hidalgoodontologia.com

ORCID: 0000-0002-4540-7715

Recibido : 03-06-2019

Aceptado: 22-07-2019 